\title{
Potential Clinical Value of 5-Hydroxytryptamine Receptor 3C as a Prognostic Biomarker for Lung Cancer
}

\author{
Jiun-Rung Chen $\mathbb{D}^{1},{ }^{1}$ Ming-Shyan Huang $\mathbb{D},{ }^{2}$ Yi-Chen Lee $\mathbb{D},{ }^{3}$ Min-His Lin, ${ }^{4}$ \\ and Yi-Fang Yang $\mathbb{1}^{5}$ \\ ${ }^{1}$ Division of Pulmonary \& Respiratory Medicine, Department of Internal Medicine, E-Da Cancer Hospital, Kaohsiung, Taiwan \\ ${ }^{2}$ Department of Internal Medicine E-Da Cancer Hospital School of Medicine, I-Shou University, Kaohsiung, Taiwan \\ ${ }^{3}$ Department of Anatomy, School of Medicine, College of Medicine, Kaohsiung Medical University, Kaohsiung, Taiwan \\ ${ }^{4}$ Division of Chest Medicine, Kaohsiung Veterans General Hospital, Kaohsiung, Taiwan \\ ${ }^{5}$ Department of Medical Education and Research, Kaohsiung Veterans General Hospital, Kaohsiung, Taiwan
}

Correspondence should be addressed to Yi-Fang Yang; yvonne845040@gmail.com

Received 11 September 2021; Accepted 30 October 2021; Published 15 November 2021

Academic Editor: Jie Mei

Copyright (C) 2021 Jiun-Rung Chen et al. This is an open access article distributed under the Creative Commons Attribution License, which permits unrestricted use, distribution, and reproduction in any medium, provided the original work is properly cited.

\begin{abstract}
Ion channels and pumps not only regulate membrane potential, ion homeostasis, and electric signaling in excitable cells but also contribute to cell proliferation, migration, apoptosis, and differentiation. Channel proteins and ion pumps can form macromolecular complexes with signaling molecules, including growth factors and cell adhesion molecules. Serotonin (5-hydroxytryptamine (5-HT)) promotes the proliferation of various cancer cell types mediated through the activation of the 5-HT receptor (HTR). Only HTR3 is a ligand-gated ion channel. However, the role of the HTR3 family of HTRs in lung cancer has not been adequately evaluated. We evaluated the relationship between the HTR3 family of HTRs and lung cancer patients' survival using Kaplan-Meier analyses and examined the expression levels of target proteins using immunohistochemistry. In this study, we found that HTR3C was amplified with high frequency in lung cancer patients, and HTR3C protein expression levels were significantly associated with lymph node metastasis and distant metastasis in lung cancer tissues. Survival analysis using the logrank test demonstrated a decrease in disease-free survival (DFS) and overall survival (OS) rates among the high-level HTR3C expression group compared with the low-level HTR3C expression group. We also evaluated the risk factors associated with lung cancer. The univariate and multivariate analyses of DFS and OS showed that HTR3C expression was a significant predictor of patient outcomes. Taken together, these data demonstrated that HTR3C expression levels were associated with poor DFS and OS in lung cancer patients, indicating that HTR3C can serve as a useful predictive biomarker for lung cancer.
\end{abstract}

\section{Introduction}

Lung cancer is a malignant tumor type and is currently the leading cause of cancer-related deaths worldwide. As high as $80 \%$ of lung cancer cases are categorized as non-small-cell lung cancer (NSCLC) $[1,2]$. Approximately $70 \%$ of newly diagnosed lung cancer patients require systemic treatment due to locally advanced or metastatic disease. However, lung cancer patients often have poor prognoses, low 5-year survival rates, and high mortality rates, most likely due to the high metastatic rate of lung cancer [2-4]. According to previous studies, ion channels contribute to the malignant behaviors of cancer cells, including migration, invasion, cell cycle control, and metastasis [5]. Ion channel upregulation and increased activity have been reported in response to mitogen exposure [6-8], and accumulating evidence supports the existence of a direct link between transmembrane ion flow and carcinogenesis $[9,10]$.

Serotonin (5-hydroxytryptamine (5-HT)) stimulates cancer cell growth in vitro [11], mediated by the activation of 5-HT receptors (HTRs, 5- $\mathrm{HT}_{1}$ to $5-\mathrm{HT}_{7}$ ). HTRs can be categorized as G protein-coupled receptors (GPCRs) and ligand-gated ion channels. Among known HTRs, only 5$\mathrm{HT}_{3}$ receptor has been identified as a ligand-gated ion channel, whereas other identified HTRs are GPCRs activated by cAMP (an intracellular messenger) [12]. The $5-\mathrm{HT}_{3}$ 
receptor family includes HTR3A, HTR3B, HTR3C, HTR3D, and HTR3E [13]. HTR3A and HTR3B assemble into 5- $\mathrm{HT}_{3}$ receptors, but HTR3C, HTR3D, and HTR3E provide molecular diversity within the family [14].

In this study, we investigated whether the $5-\mathrm{HT}_{3}$ ionotropic receptor family was associated with a malignant lung cancer phenotype. We examined the genetic variations in members of the HTR3 family in NSCLC patient data contained in a dataset obtained from The Cancer Genome Atlas (TCGA) and evaluated the relationship between HTR3 family mutations and patient survival using a Kaplan-Meier analysis. Based on bioinformatics analysis, we identified targets induced by HTR3 activation and examined relationships between these protein expression levels and clinical outcomes in patients with lung cancer.

\section{Materials and Methods}

2.1. In Silico Genetic Profiles and Kaplan-Meier Analysis of HTR3 Family Members. Genetic variations in HTR3 family members and mutation frequency were analyzed using the online TCGA dataset (cBioPortal, https://www. cbioportal.org/). Kaplan-Meier analysis (overall survival (OS)) was examined using a lung cancer microarray dataset [15].

2.2. Specimens. Lung cancer patients were recruited from E-Da Hospital after obtaining IRB approval (EMRP-107069). All specimens were subjected to immunohistochemical staining to evaluate the expression of HTR3C. Histological grade was classified according to the recommendations of the World Health Organization (WHO). Histological diagnostic features, including tumor size, local invasion, lymph node involvement, distant metastasis, and final disease stage, were determined according to the American Joint Committee on Cancer (AJCC) tumor node metastasis (TNM) classification system for lung cancer [2]. Follow-up lasted up to 200 months.

2.3. Immunohistochemistry. Immunohistochemistry (IHC) was performed according to the manufacturer's instructions. In brief, tissue slides were deparaffinized, rehydrated with a graded ethanol solution, and then rinsed with distilled water. Antigen retrieval was performed by immersing the slides in retrieval solution (pH6.0) for $15 \mathrm{~min}$ at $96^{\circ} \mathrm{C}$. The tissue slides were incubated with primary antibody against HTR3C (1:50; \#ab199148; Abcam, Cambridge, United Kingdom) for $30 \mathrm{~min}$ at room temperature. The antigen-antibody complexes were detected by the avidin-biotin-peroxidase method (using $3,3^{\prime}$-diaminobenzidine as a chromogenic substrate). A negative control incubated immunoglobulin fraction of nonimmune rabbit serum for each staining. HTR3C expression was classified according to the $\mathrm{H}$-score, which was calculated as the percentage of positively stained cells multiplied by the staining intensity.
2.4. Statistical Analysis. All statistical analyses were performed using SPSS 19.0 statistical package. Associations between HTR3 or downstream targets and tumor stage, tumor grade, age at diagnosis, tumor size, lymph nodes status, and recurrence were analyzed using the two-sided $\chi^{2}$ test. Survival curves were generated using Kaplan-Meier estimates, and significant differences between curves were evaluated using the two-sided log-rank test. Hazard ratios (HR) and 95\% confidence intervals (CIs) were computed for univariate and multivariate Cox regression models to investigate the correlation between clinicopathological characteristics and survival. $P<0.05$ was considered significant.

\section{Results}

3.1. In Silico Genetic Profiles of HTR3 Family Members in NSCLC. First, we evaluated the genetic profiles of HTR3 family members (HTR3A-HTR3E) in NSCLC patients using a publicly available dataset (cBioPortal). The genetic profiles of HTR3 family members were obtained from 6,122 NSCLC samples, which revealed that $H T R 3 C, H T R 3 D$, and HTR $3 E$ were significantly amplified in NSCLC genetic profiles (Figure 1). We next analyzed the frequency of gene amplification co-occurrence within the same samples from lung cancer patients, which showed high frequencies of co-occurrence for some gene amplification pairs within the same patient, including HTR3C/HTR3D, HTR3C/HTR $3 E$, and HTR3D/HTR3E, whereas the co-occurrence of gene amplification was not observed for other gene pairs (HTR3A/ HTR $3 B$, HTR $3 B / H T R 3 D$, and HTR3B/HTR3C) (Supplementary Table 1).

3.2. High HTR3C Expression Levels in Tumor Tissues Are Associated with Poor Clinical Outcomes in Patients with NSCLC. To evaluate which members of the HTR3 family were associated with lung cancer progression, we used gene expression microarray analysis, followed by Kaplan-Meier survival curve analysis, which showed that HTR $3 A, H T R 3 B$, and HTR3C expression levels were associated with poor OS (Figures 2(a)-2(c)). The results of the survival analysis suggested that the HTR3 family plays important roles in lung cancer progression. We evaluated the direct interaction partners of HTR3C using the BioGRID website and found that inositol monophosphatase domain-containing 1 (IMPAD1) interacts with HTR3C (Supplementary Table 2). IMPAD1 acts as a mitochondrial electron transport inhibitor and promotes lung cancer metastasis [16]. We further examined the correlation between HTR $3 C$ and IMPAD1 mRNA levels, which showed that HTR3C mRNA levels were positively correlated with IMPAD1 mRNA levels in lung cancer tissues (GSE31210; Figure 2(d)). The combination of HTR3C and IMPAD1 expression was further evaluated by comparing lung cancer patients with high $H T R 3 C$ and high IMPAD1 expression against patients with low HTR3C and low IMPAD1 expression using the Kaplan-Meier plotter website [17]. The high HTR3C/high IMPAD1 expression group was associated with shorter OS than the low HTR3C/low IMPAD1 expression group among lung cancer patients (Figure 2(e)). 


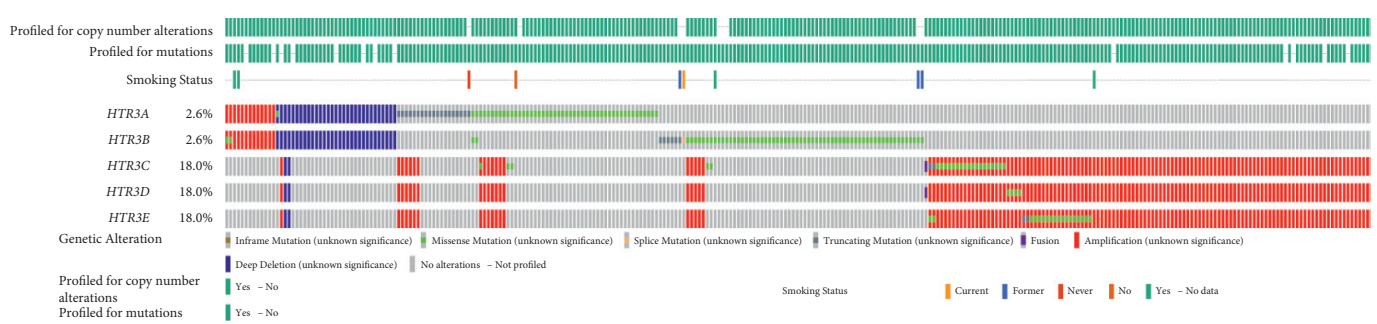

(a)
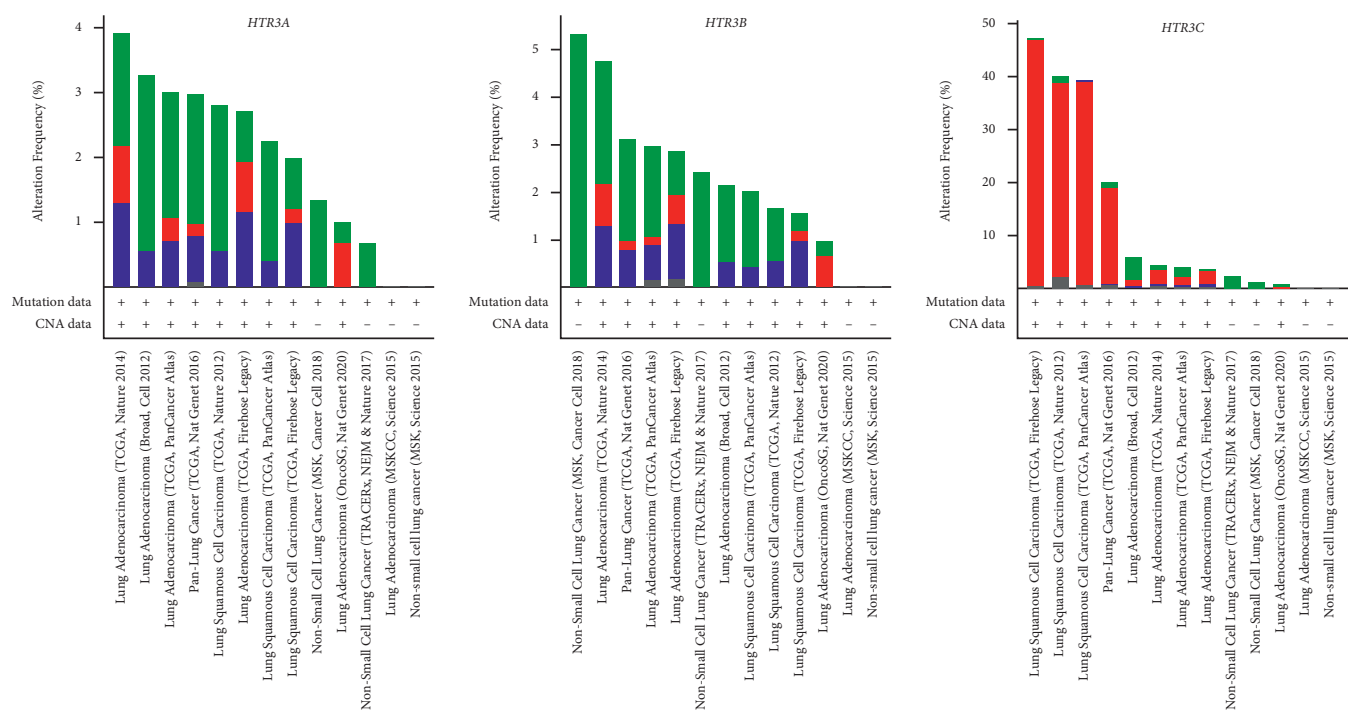

- Mutation

- Amplification

- Mutation

- Amplification

- Mutation

- Deep Deletion

- Deep Deletion

(b)

(c)

plification

- Deep Deletion

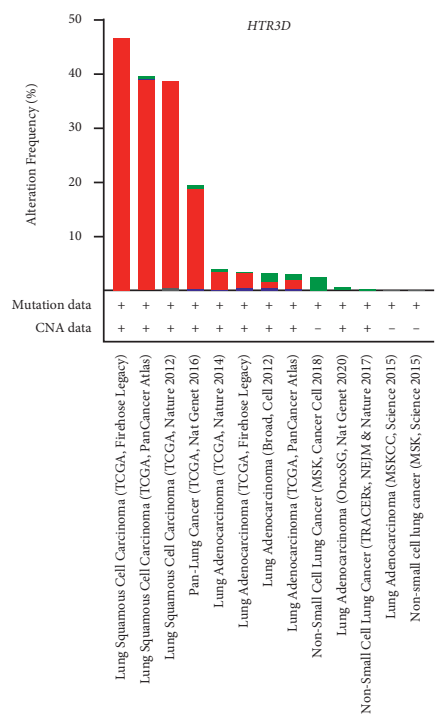

- Mutation

- Amplification

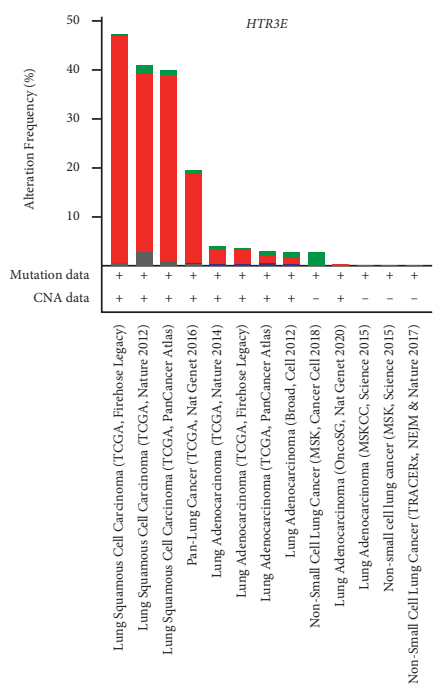

- Mutation

- Deep Deletion

(d)

(e)

(f)

FIgURE 1: HTR3C, HTR3D, and HTR3E showed the highest amplification levels in NSCLC. (a) OncoPrint showing HTR3C, HTR3D, and HTR $3 E$ amplification in $18 \%$ of NSCLC cases. Colors indicate the type of genetic mutation (missense, in-frame, truncated, amplification, deletion, and fusion), and different cohorts are listed below the OncoPrint. Cancer type summary of HTR3A (b), HTR3B (c), HTR3C (d), HTR3D (e), and HTR3E (f) according to the lung cancer cohorts (cBioPortal). CAN = copy number alterations. 

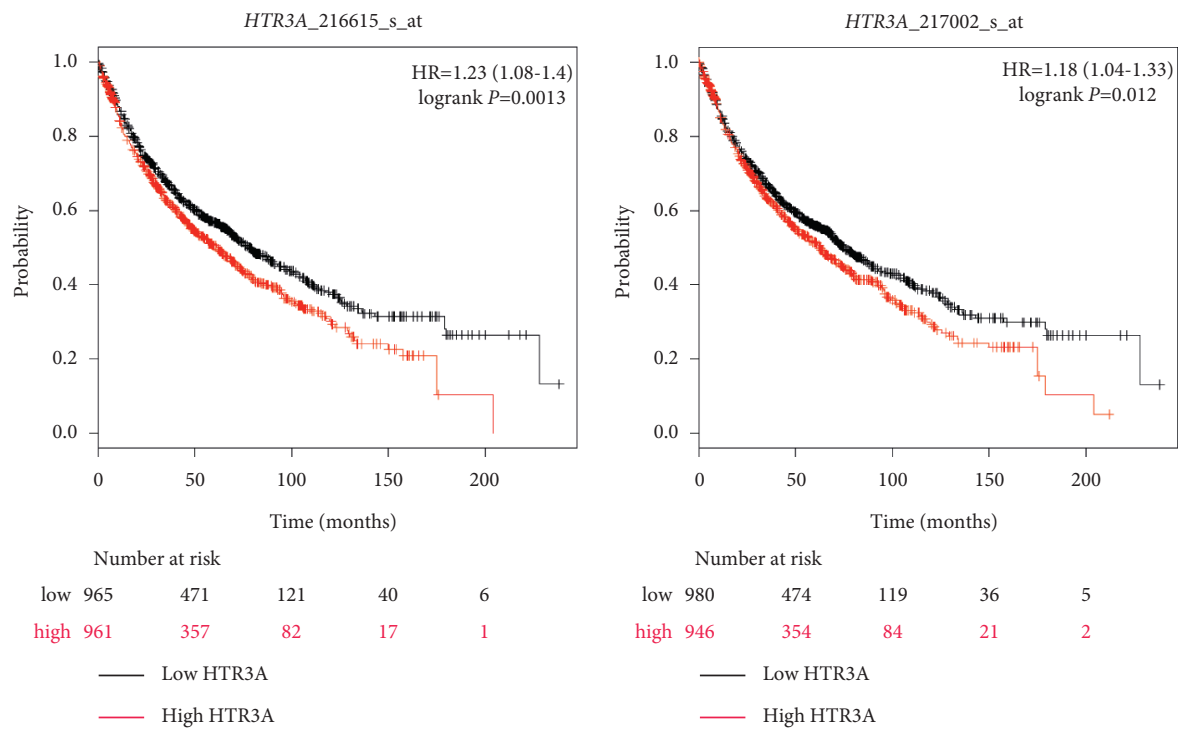

_ Low HTR3A

(a)
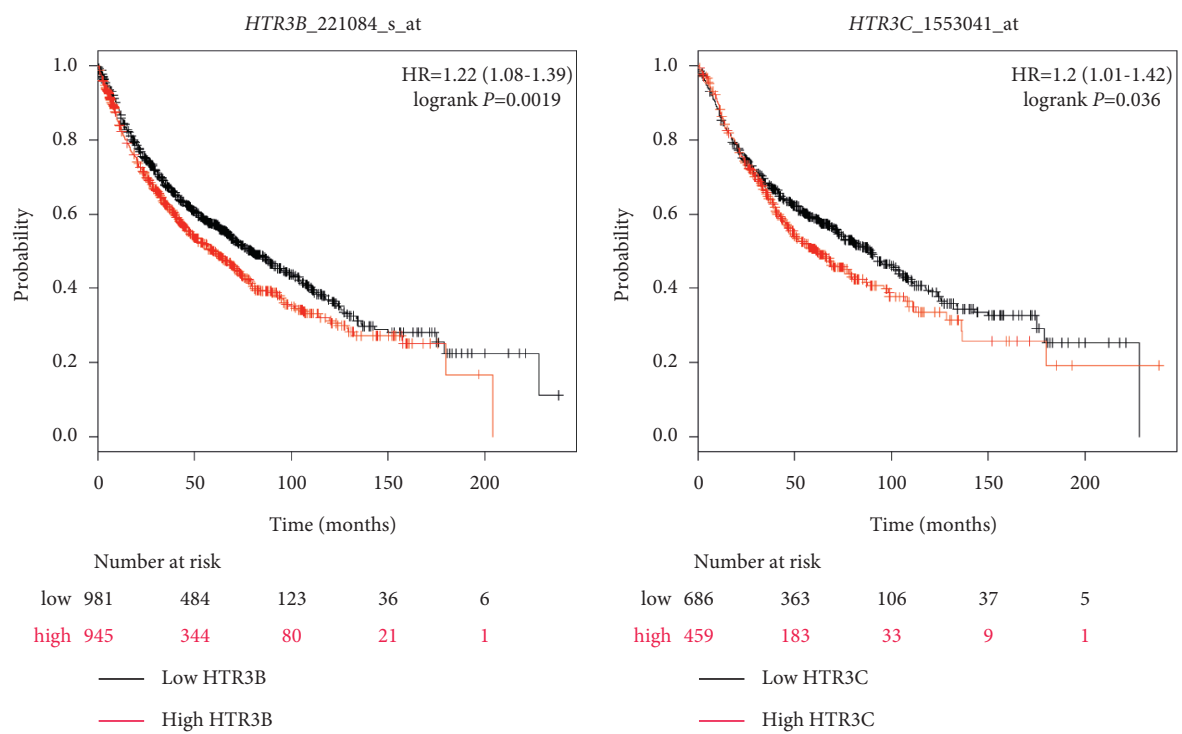

(b)

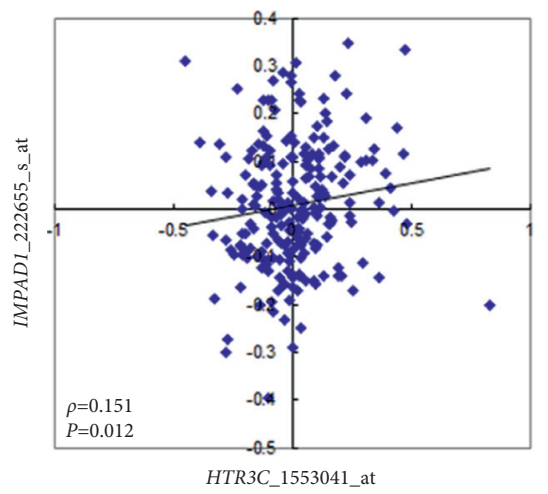

(c)

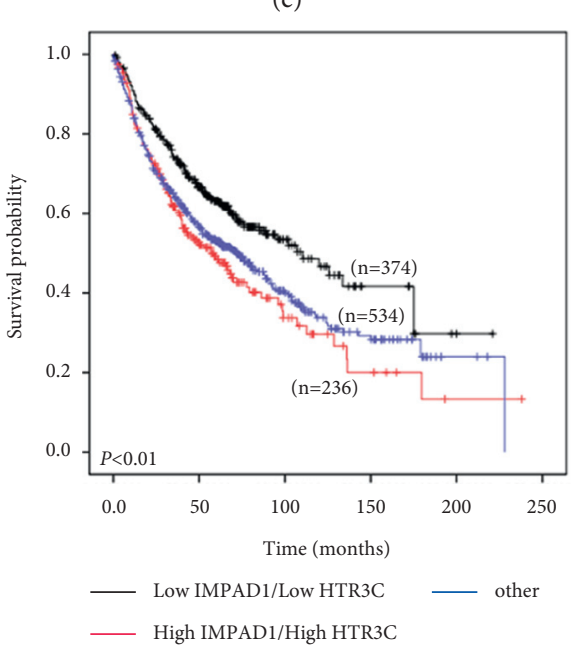

(d)

(e)

FIgURe 2: HTR3 family as a potential target for the prevention of lung cancer progression. Kaplan-Meier curve for overall survival, according to the mRNA expression HTR3A (a), HTR3B (b), and HTR3C (c), using publicly available lung cancer microarray datasets. $\mathrm{HR}=$ hazard ratio. (d) Analysis correlation of HTR3C and IMPAD1 mRNA expression by using microarray datasets of lung cancer (NCBI/ GEO/GSE31210). (e) Relative mRNA expression levels of HTR3C and IMPAD1 in lung cancer patients (online KM plotter). 

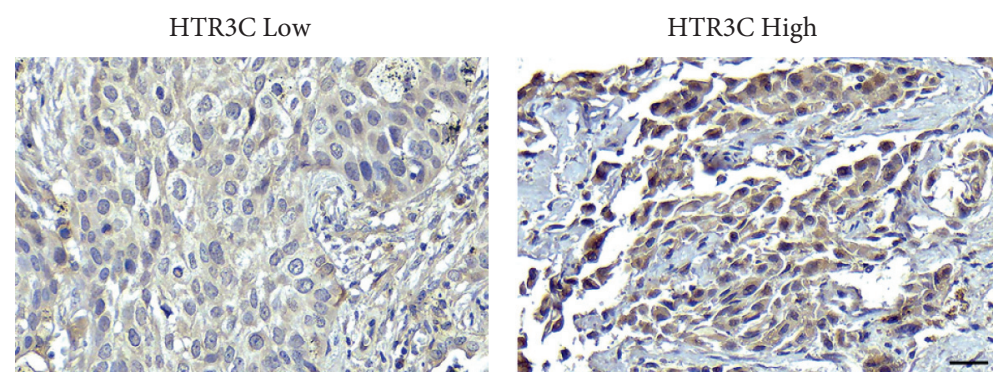

(a)
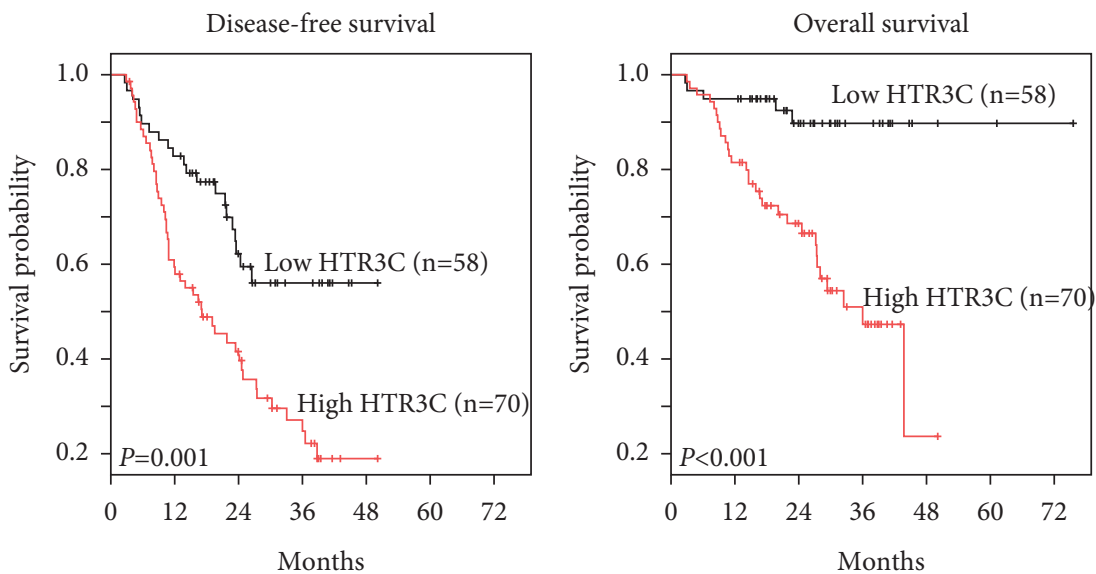

(b)
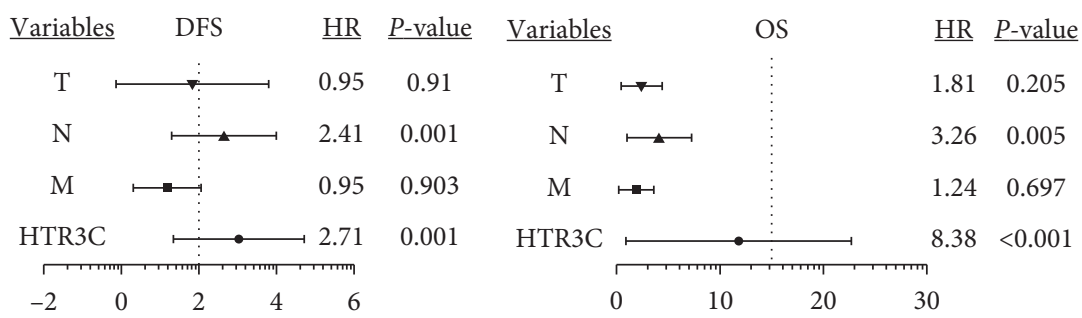

(c)

FIGURE 3: HTR3C is overexpressed in lung cancer and correlates with worse survival. (a) Representative images of HTR3C IHC staining in lung cancer tissues. (b) Kaplan-Meier curve for disease-free survival (DFS) and overall survival (OS) among lung cancer patients according to HTR3C expression. (c) Hazard ratios were determined by multivariate Cox regression analysis.

Moreover, $H T R 3 C$ gene amplification correlated with $H T R 3 C$ mRNA expression in lung cancer patients (Supplementary Figure 1). Therefore, we further examined the characteristics of HTR3C and its contributions to lung cancer.

3.3. HTR3C Protein Upregulation Is Associated with Poor Clinical Outcomes in Lung Cancer. To evaluate HTR3C protein levels in lung cancer, we examined the expression of HTR3C in primary lung tumors from 128 patients using IHC and correlated HTR3C expression with the clinicopathological characteristics of patients. As shown in Figure 3(a), HTR3C protein expression in cancer tissues was classified into low and high expression level groups. We found that high HTR3C expression levels in lung cancer tissues were significantly associated with positive lymph node metastasis ( $\mathrm{N}$ status; $P=0.028$ ), positive distant metastasis (M status; $P=0.042)$, recurrence $(P<0.001)$, and cancer death $(P<0.001$; Table 1). Survival analysis using the log-rank test demonstrated decreased disease-free survival (DFS) and OS rates for the high HTR3C expression level group compared with those for the low HTR3C expression level group $(P<0.01$; Figure $3(\mathrm{~b}))$. We also evaluated the risk factors associated with lung cancer. Univariate and multivariable Cox regression analyses were used to estimate HRs, which showed that HTR3C expression level was a significant predictor of both OS and DFS (Figure 3(c), Tables 2 and 3).

\subsection{HTR3C Expression Positively Correlates with TTN in Lung} Cancer Patients. To evaluate whether the genetic alteration events reflect the HTR3C expression level in lung cancer patients, we screened and listed the most commonly mutated gene in lung cancer patients. Our results indicated that these genes' (TP53, TTN, MUC16, RYR2, CSMD3, USH2A, $L R P 1 B, Z F H X 4$, SYNE1, and XIRP2) mutations are 


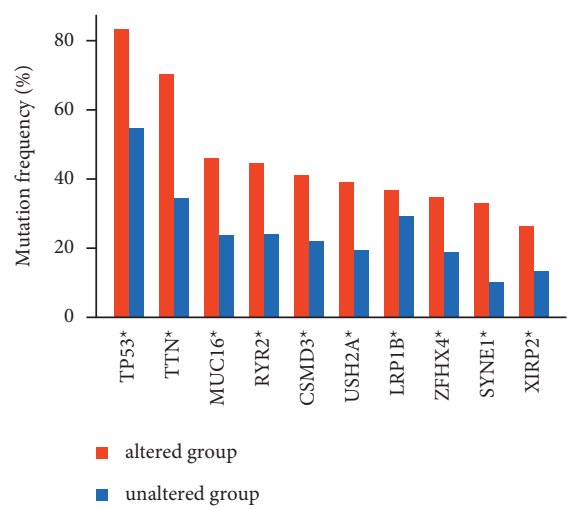

(a)

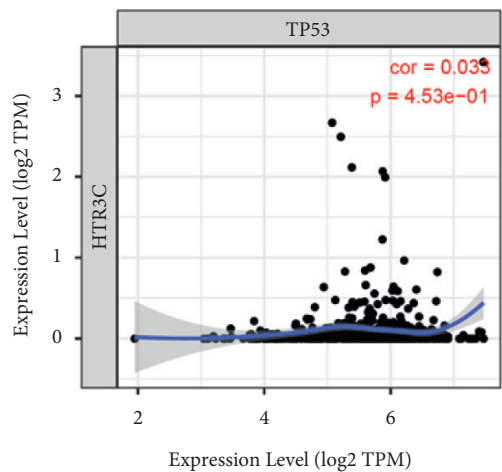

Expression Level ( $\log 2$ TPM)

\begin{tabular}{cccccccccc}
\hline \multirow{2}{*}{ A } & \multirow{2}{*}{ B } & Neither & A Not B & B Not A & Both & $\begin{array}{c}\text { Log2 Odds } \\
\text { Ratio }\end{array}$ & p-Value & q-Value Tendency \\
\hline HTR3C & TP53 & 1429 & 102 & 1930 & 552 & 2.003 & $<0.001$ & $<0.001$ & Co-occurrence \\
HTR3C & TTN & 1675 & 170 & 1684 & 484 & 1.502 & $<0.001$ & $<0.001$ & Co-occurrence \\
HTR3C & MUC16 & 2165 & 379 & 1194 & 275 & 0.396 & $<0.001$ & $<0.001$ & Co-occurrence \\
\hline
\end{tabular}

(b)

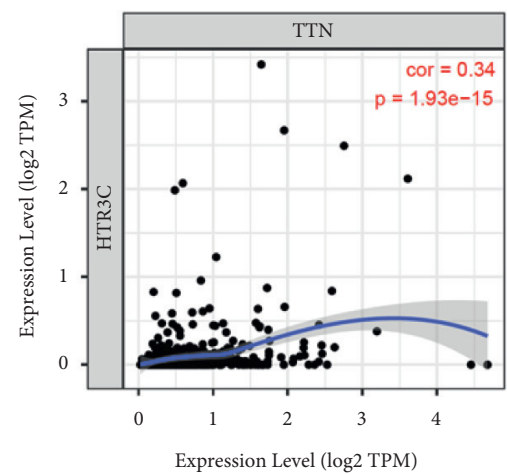

(c)

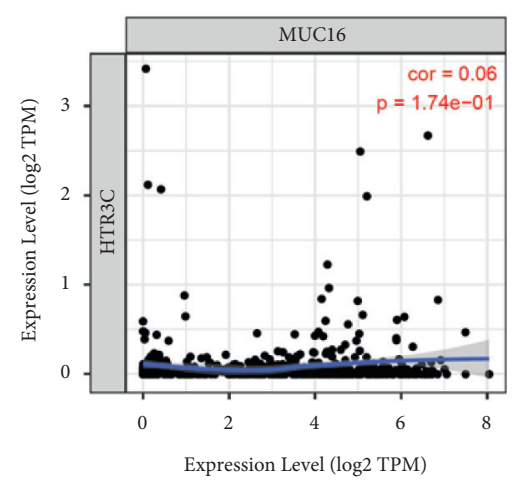

FIGURE 4: Correlation analysis between HTR3C and oncogene expression in lung cancer patients. (a) Frequency of genomic alterations in tumors obtained from lung cancer patients. ${ }^{*} P<0.05$. (b) Table of major co-occurring genomic alterations in HTR3C-amplified NSCLC. (c) Analysis of the correlations between HTR3C and TP53/TTN/MUC16 mRNA expression levels using TIMER.

frequently present in clinical lung cancer patients (Figure 4(a)). We focus on top three genes; Figure 4(b) shows the co-occurrence between HTR3C gene alterations and a series of core lung cancer-associated genes, including TP53, TTN, and MUC16. We further examined the correlation between the mRNA expression levels of HTR3C and those of TP53, TTN, and MUC16 using TIMER [18]. The results showed that $H T R 3 C$ mRNA levels were positively correlated with TTN mRNA levels in lung cancer tissues (Figure 4(c)).

\section{Discussion}

In this study, HTR3 family members were assessed in NSCLC patients, which showed that HTR3C, HTR3D, and HTR3E were amplified with high frequency among lung cancer patients. HTR $3 A, H T R 3 B$, and HTR $3 C$ mRNA expression levels were associated with poor OS. Based on the IHC results, high protein expression levels of HTR3C were associated with lymph node metastasis, distant metastasis, and recurrence in lung cancer patients. Moreover, lung cancer patients presenting with high HTR3C protein levels were associated with shorter OS and DFS than patients with low HTR3C protein levels. TP53, TNT, and MUC16 mutations occurred at high frequencies among NSCLC patients and co-occurrence with altered HTR3C. HTR3C mRNA levels were positively correlated with TNT mRNA levels in lung cancer tissues. These findings suggest that HTR3C may serve as a prognostic marker in lung cancer.

5-HT can affect tumor behaviors via the activation of HTRs. In vitro studies have shown that 5-HT promotes cell growth in cancer cell lines, including small-cell lung cancer (SCLC) cells, mediated by the activation of the HTRs 5- $\mathrm{HT}_{1 \mathrm{~A}}$ and $5-\mathrm{HT}_{1 \mathrm{D}}[11,19]$. The proliferation of endothelial cells is stimulated by the activation of the HTRs $5-\mathrm{HT}_{1}, 5-\mathrm{HT}_{2}$, and $5-\mathrm{HT}_{3}$, and $5-\mathrm{HT}_{2}$ activation was shown to mediate similar effects in aortic smooth muscle cells [20]. 5-HT activates mitogen-activated protein kinase (MAPK) and the phosphoinositide 3-kinase (PI3K)/protein kinase $\mathrm{B}$ (AKT) signaling pathway to promote malignant behaviors in prostate cancer cells [21] through several receptor subtypes, promoting disease progression in prostate cancer patients $[11,22]$. Wang et al. showed that the HTRs $5-\mathrm{HT}_{3}$ and $5-\mathrm{HT}_{4}$, which are located on the mitochondria membrane, modulate the respiration control ratio (RCR) in cardiac mitochondria. Moreover, HTR3 has been reported to regulate mitochondrial functions, altering ATP contents and lactate dehydrogenase (LDH) release [23]. Our data showed that HTR3C expression was significantly associated with lymph node metastasis, distant metastasis, and recurrence in lung cancer patients. In the BioGRID dataset, HTR3C interaction with the oncogene IMPAD1 was shown to promote lung cancer metastasis via the inhibition of mitochondrial electron transport [16]. The high HTR3C/high IMPAD1 expression cohort showed a 
TABLE 1: Correlation between HTR3C expression levels and various clinicopathological characteristics in lung cancer.

\begin{tabular}{|c|c|c|c|c|c|c|c|}
\hline \multirow{3}{*}{ Variables } & \multirow{3}{*}{ Item } & \multirow{3}{*}{ Patient no. (\%) } & \multicolumn{4}{|c|}{ HTR3C } & \multirow{3}{*}{$P$ value } \\
\hline & & & \multicolumn{2}{|c|}{ Low $(\leq 92.62)$} & \multicolumn{2}{|c|}{ High (>92.62) } & \\
\hline & & & No. & $\%$ & No. & $\%$ & \\
\hline \multirow{3}{*}{ Age (y) } & & $128(100.0)$ & 58 & 45.3 & 70 & 54.7 & \\
\hline & $\leq 70$ & $98(76.6)$ & 41 & 70.7 & 57 & 81.4 & $0.153^{\mathrm{a}}$ \\
\hline & $>70$ & $30(23.4)$ & 17 & 29.3 & 13 & 18.6 & \\
\hline \multirow{2}{*}{ Sex } & Female & $46(35.9)$ & 18 & 31.0 & 28 & 40.0 & $0.293^{\mathrm{a}}$ \\
\hline & Male & $82(64.1)$ & 40 & 69.0 & 42 & 60.0 & \\
\hline \multirow{2}{*}{ Grade } & I & $29(22.7)$ & 10 & 17.2 & 19 & 27.1 & $0.183^{\mathrm{a}}$ \\
\hline & II/III & $99(77.3)$ & 48 & 82.8 & 51 & 72.9 & \\
\hline \multirow{2}{*}{ T status } & $\mathrm{T} 1 / \mathrm{T} 2$ & $107(83.6)$ & 45 & 77.6 & 62 & 88.6 & $0.095^{\mathrm{a}}$ \\
\hline & $\mathrm{T} 3 / \mathrm{T} 4$ & $21(16.4)$ & 13 & 22.4 & 8 & 11.4 & \\
\hline \multirow{2}{*}{$\mathrm{N}$ status } & Negative (N0) & $68(53.1)$ & 37 & 63.8 & 31 & 44.3 & $0.028^{\mathrm{a}}$ \\
\hline & Positive (N1/N2/N3) & $60(46.9)$ & 21 & 36.2 & 39 & 55.7 & \\
\hline \multirow{2}{*}{ M status } & Negative & $110(85.9)$ & 54 & 90.2 & 56 & 76.6 & $0.042^{\mathrm{b}}$ \\
\hline & Positive & $18(14.1)$ & 4 & 9.8 & 14 & 23.4 & \\
\hline \multirow{2}{*}{ Histology } & Adenocarcinoma & $97(78.2)$ & 43 & 75.4 & 54 & 80.6 & $0.488^{\mathrm{a}}$ \\
\hline & Squamous cell carcinoma & $27(21.8)$ & 14 & 24.6 & 13 & 19.4 & \\
\hline \multirow{2}{*}{ Performance } & 0 & $85(66.4)$ & 40 & 69.0 & 45 & 64.3 & $0.577^{\mathrm{a}}$ \\
\hline & 1 & $43(33.6)$ & 18 & 31.0 & 25 & 35.7 & \\
\hline \multirow{3}{*}{ Smoking status } & Never & $76(59.4)$ & 34 & 58.6 & 42 & 60.0 & $0.472^{\mathrm{a}}$ \\
\hline & Former & $22(17.2)$ & 8 & 13.8 & 14 & 20.0 & \\
\hline & Current & $30(23.4)$ & 16 & 27.6 & 14 & 20.0 & \\
\hline \multirow{2}{*}{ Recurrence } & No & $58(45.3)$ & 37 & 63.8 & 21 & 30.0 & $<0.001^{\mathrm{a}}$ \\
\hline & Yes & $70(54.7)$ & 21 & 36.2 & 49 & 70.0 & \\
\hline \multirow{2}{*}{ Cancer death } & No & $93(72.7)$ & 53 & 91.4 & 40 & 57.1 & $<0.001^{\mathrm{b}}$ \\
\hline & Yes & $35(27.3)$ & 5 & 8.6 & 30 & 42.9 & \\
\hline
\end{tabular}

${ }^{\mathrm{a}} P$ value was calculated by the chi-square test. ${ }^{\mathrm{b}} P$ value was calculated by Fisher's exact test.

TABLE 2: Univariate and multivariate analysis of overall survival for lung cancer.

\begin{tabular}{|c|c|c|c|c|c|c|c|}
\hline \multirow{2}{*}{ Variables } & \multirow{2}{*}{ Item } & \multicolumn{3}{|c|}{ Univariate } & \multicolumn{3}{|c|}{ Multivariate } \\
\hline & & HR & $95 \%$ CI & $P$ value & HR & $95 \% \mathrm{CI}$ & $P$ value \\
\hline \multirow{2}{*}{ Age (y) } & $>70$ & 1.62 & $(0.79,3.30)$ & 0.188 & 2.37 & $(0.98,5.73)$ & 0.056 \\
\hline & $\leq 70$ & 1.00 & & & 1.00 & & \\
\hline \multirow{2}{*}{ Sex } & Male & 1.50 & $(0.70,3.21)$ & 0.295 & 0.74 & $(0.26,2.11)$ & 0.576 \\
\hline & Female & 1.00 & & & 1.00 & & \\
\hline \multirow{2}{*}{ Grade } & II/III & 1.82 & $(0.70,4.68)$ & 0.218 & 1.05 & $(0.33,3.33)$ & 0.928 \\
\hline & I & 1.00 & & & 1.00 & & \\
\hline \multirow{2}{*}{ T status } & T4/T3 & 1.44 & $(0.65,3.18)$ & 0.364 & 1.81 & $(0.72,4.52)$ & 0.205 \\
\hline & $\mathrm{T} 2 / \mathrm{T} 1$ & 1.00 & & & 1.00 & & \\
\hline \multirow{2}{*}{$\mathrm{N}$ status } & Positive & 3.43 & $(1.64,7.19)$ & 0.001 & 3.26 & $(1.42,7.48)$ & 0.005 \\
\hline & Negative & 1.00 & & & 1.00 & & \\
\hline \multirow{2}{*}{ M status } & Positive & 3.35 & $(1.59,7.08)$ & 0.002 & 1.24 & $(0.42,3.73)$ & 0.697 \\
\hline & Negative & 1.00 & & & 1.00 & & \\
\hline \multirow{2}{*}{ Histology } & Squamous cell carcinoma & 2.72 & $(1.36,5.44)$ & 0.005 & 3.98 & $(1.59,9.94)$ & 0.003 \\
\hline & Adenocarcinoma & 1.00 & & & 1.00 & & \\
\hline \multirow{2}{*}{ Performance } & 1 & 4.55 & $(2.28,9.07)$ & $<0.001$ & 5.34 & $(2.24,12.73)$ & $<0.001$ \\
\hline & 0 & 1.00 & & & 1.00 & & \\
\hline \multirow{3}{*}{ Smoking status } & Current & 2.54 & $(1.19,5.41)$ & 0.016 & 1.71 & $(0.56,5.21)$ & 0.345 \\
\hline & Former & 1.89 & $(0.79,4.52)$ & 0.152 & 2.09 & $(0.70,6.26)$ & 0.187 \\
\hline & Never & 1.00 & & & 1.00 & & \\
\hline \multirow{2}{*}{ HTR3C } & High & 5.69 & $(2.20,14.73)$ & $<0.001$ & 8.38 & $(2.93,23.98)$ & $<0.001$ \\
\hline & Low & 1.00 & & & 1.00 & & \\
\hline
\end{tabular}

$\mathrm{HR}=$ hazard ratio $\mathrm{CI}=$ confidence interval. 
TABLE 3: Univariate and multivariate analysis of disease-free survival for lung cancer.

\begin{tabular}{|c|c|c|c|c|c|c|c|}
\hline \multirow{2}{*}{ Variables } & \multirow{2}{*}{ Item } & \multicolumn{3}{|c|}{ Univariate } & \multicolumn{3}{|c|}{ Multivariate } \\
\hline & & HR & $95 \%$ CI & $P$ value & HR & $95 \% \mathrm{CI}$ & $P$ value \\
\hline \multirow{2}{*}{ Age (y) } & $>70$ & 1.22 & $(0.71,2.12)$ & 0.471 & 1.29 & $(0.68,2.42)$ & 0.438 \\
\hline & $\leq 70$ & 1.00 & & & 1.00 & & \\
\hline \multirow{2}{*}{ Sex } & Male & 0.90 & $(0.55,1.47)$ & 0.680 & 0.88 & $(0.48,1.61)$ & 0.677 \\
\hline & Female & 1.00 & & & 1.00 & & \\
\hline \multirow{2}{*}{ Grade } & II/III & 2.04 & $(0.52,1.81)$ & 0.037 & 1.87 & $(0.88,4.00)$ & 0.105 \\
\hline & I & 1.00 & & & 1.00 & & \\
\hline \multirow{2}{*}{$\mathrm{T}$ status } & T4/T3 & 0.97 & $(0.51,1.64)$ & 0.919 & 0.95 & $(0.45,2.03)$ & 0.901 \\
\hline & $\mathrm{T} 2 / \mathrm{T} 1$ & 1.00 & & & 1.00 & & \\
\hline \multirow{2}{*}{$\mathrm{N}$ status } & Positive & 2.98 & $(1.81,4.90)$ & $<0.001$ & 2.41 & $(1.42,4.08)$ & 0.001 \\
\hline & Negative & 1.00 & & & 1.00 & & \\
\hline \multirow{2}{*}{ M status } & Positive & 2.41 & $(1.37,4.23)$ & 0.002 & 0.95 & $(0.43,2.13)$ & 0.903 \\
\hline & Negative & 1.00 & & & 1.00 & & \\
\hline \multirow{2}{*}{ Histology } & Squamous cell carcinoma & 1.73 & $(1.02,2.96)$ & 0.043 & 1.94 & $(1.03,3.65)$ & 0.041 \\
\hline & Adenocarcinoma & 1.00 & & & 1.00 & & \\
\hline \multirow{2}{*}{ Performance } & 1 & 2.24 & $(1.40,3.58)$ & 0.001 & 2.16 & $(1.18,3.94)$ & 0.012 \\
\hline & 0 & 1.00 & & & 1.00 & & \\
\hline \multirow{3}{*}{ Smoking status } & Current & 1.06 & $(0.60,1.90)$ & 0.835 & 1.08 & $(0.49,2.37)$ & 0.856 \\
\hline & Former & 1.07 & $(0.58,1.97)$ & 0.840 & 1.18 & $(0.57,2.45)$ & 0.654 \\
\hline & Never & 1.00 & & & 1.00 & & \\
\hline \multirow{2}{*}{ HTR3C } & High & 2.37 & $(1.42,3.96)$ & 0.001 & 2.71 & $(1.51,4.85)$ & 0.001 \\
\hline & Low & 1.00 & & & 1.00 & & \\
\hline
\end{tabular}

$\mathrm{HR}=$ hazard ratio; $\mathrm{CI}=$ confidence interval.

significant decrease in OS compared with the low HTR3C/low IMPAD1 expression cohort (Figure 2(e)). These findings suggested that the HTR3C/IMPAD1 axis may play a role in lung cancer progression.

Previously, programmed cell death ligand (PD-L1) expression as detected by IHC was designated as the first Food and Drug Administration (FDA) approved biomarker for the monitoring of patient's responsiveness to immune checkpoint blockade (ICB) immunotherapy. In addition, several studies have shown that tumor mutational burden (TMB) can serve as a biomarker for predicting the responsiveness to ICB-based immunotherapy for a variety of tumor types $[24,25]$. The DNA damage response (DDR) pathway [26], deficient mismatch repair (dMMR) [27], TP53/KRAS comutation [28], and TTN (Titin) mutations [29] have also been established as predictive biomarkers for ICB treatment. Our study revealed that TP53, TNT, and MUC16 mutations were co-occurrence with altered HTR3C in NSCLC patients. Furthermore, HTR3C mRNA expression levels were correlated with TNT mRNA expression levels in NSCLC patients. We could not exclude the possibility that HTR3C amplification or expression may also be correlated with the response rate to ICB; however, this is the first study to evaluate alterations in the HTR3C gene and protein expression in lung cancer patients.

This is the first study to evaluate genetic variations in the HTR3 family, which revealed HTR3C, HTR3D, and HTR3E gene amplification in NSCLC patients. High levels of HTR3C protein expression were correlated with shorter OS and DFS compared with low levels of HTR3C protein expression, and Cox regression analyses demonstrated that
HTR3C expression levels could serve as an independent prognostic factor for lung cancer outcomes.

\section{Data Availability}

The datasets supporting the conclusions of this article are included within the article.

\section{Ethical Approval}

This study was approved by the E-Da Hospital, after obtaining IRB approval (EMRP-107-069).

\section{Conflicts of Interest}

The authors declare that they have no conflicts of interest.

\section{Authors' Contributions}

Jiun-Rung Chen and Ming-Shyan Huang contributed equally to this work. J.-R C and Y.-F.Y designed and wrote the manuscript. J.-R C and M.-S H provided materials. Y.-C. L and Y.-F. Y performed experiments. J.-R C, M.-H L, Y.-C. $\mathrm{L}$, and Y.-F. Y interpreted data.

\section{Acknowledgments}

This study was supported by grants from the E-Da Cancer Hospital (grant no. EDCHC-108001), Kaohsiung Veterans General Hospital, Taiwan (grant no. KSVGH110-144), and National Science Council (MOST 110-2314B-075B-009MY3). 


\section{Supplementary Materials}

Figure S1: HTR3C amplification and expression levels in NSCLC as reported by cBioPortal. Table S1: HTR3 family members' mutual exclusivity. Table S2: displaying 121 total unique interactors of HTR3C. (Supplementary Materials)

\section{References}

[1] D. C. Ihde, "Chemotherapy of lung cancer," New England Journal of Medicine, vol. 327, no. 20, pp. 1434-1441, 1992.

[2] P. C. Hoffman, A. M. Mauer, and E. E. Vokes, "Lung cancer," The Lancet, vol. 355, no. 9202, pp. 479-485, 2000.

[3] A. Spira and D. S. Ettinger, "Multidisciplinary management of lung cancer," New England Journal of Medicine, vol. 350, no. 4, pp. 379-392, 2004.

[4] R. S. Herbst, J. V. Heymach, and S. M. Lippman, "Lung cancer," New England Journal of Medicine, vol. 359, no. 13, pp. 1367-1380, 2008.

[5] A. Litan and S. A. Langhans, "Cancer as a channelopathy: ion channels and pumps in tumor development and progression," Frontiers in Cellular Neuroscience, vol. 9, p. 86, 2015.

[6] T. M. Campbell, M. J. Main, and E. M. Fitzgerald, "Functional expression of the voltage-gated $\mathrm{Na}(+)$-channel Nav1.7 is necessary for EGF-mediated invasion in human non-small cell lung cancer cells," Journal of Cell Science, vol. 126, no. Pt 21, pp. 4939-4949, 2013.

[7] K. Enomoto, M. F. Cossu, T. Maeno, C. Edwards, and T. Oka, "Involvement of the $\mathrm{Ca} 2+$-dependent $\mathrm{K}+$ channel activity in the hyperpolarizing response induced by epidermal growth factor in mammary epithelial cells," FEBS Letters, vol. 203, no. 2, pp. 181-184, 1986.

[8] J. Frede, S. P. Fraser, G. Oskay-Özcelik et al., "Ovarian cancer: ion channel and aquaporin expression as novel targets of clinical potential," European Journal of Cancer, vol. 49, no. 10, pp. 2331-2344, 2013.

[9] C. D. Cone Jr., "Electroosmotic interactions accompanying mitosis initation in sarcoma cells in vitro," Transactions of the New York Academy of Science, vol. 31, no. 4, pp. 404-427, 1969.

[10] C. D. Cone Jr., "Unified theory on the basic mechanism of normal mitotic control and oncogenesis," Journal of Theoretical Biology, vol. 30, no. 1, pp. 151-181, 1971.

[11] D. Sarrouilhe, J. Clarhaut, N. Defamie, and M. Mesnil, "Serotonin and cancer: what is the link?" Current Molecular Medicine, vol. 15, no. 1, pp. 62-77, 2015.

[12] J. F. Marwood and G. S. Stokes, "Serotonin (5Ht) and its antagonists: involvement in the cardiov ascular system," Clinical and Experimental Pharmacology and Physiology, vol. 11, no. 5, pp. 439-455, 1984.

[13] B. Niesler, B. Frank, J. Kapeller, and G. A. Rappold, "Cloning, physical mapping and expression analysis of the human 5HT3 serotonin receptor-like genes HTR3C, HTR3D and HTR3E," Gene, vol. 310, pp. 101-111, 2003.

[14] N. M. Barnes, T. G. Hales, S. C. R. Lummis, and J. A. Peters, "The 5-HT3 receptor-the relationship between structure and function," Neuropharmacology, vol. 56, no. 1, pp. 273-284, 2009.

[15] Á. Nagy, G. Győrffy, and B. Gyorffy, "Pancancer survival analysis of cancer hallmark genes," Scientific Reports, vol. 11, no. 1, p. 6047, 2021.

[16] Y.-F. Yang, Y.-Y. Wang, M. Hsiao et al., "IMPAD1 functions as mitochondrial electron transport inhibitor that prevents ROS production and promotes lung cancer metastasis through the AMPK-Notch1-HEY1 pathway," Cancer Letters, vol. 485 , pp. 27-37, 2020.

[17] B. Gyorffy, P. Surowiak, J. Budczies, and A. Lanczky, "Online survival analysis software to assess the prognostic value of biomarkers using transcriptomic data in nonsmall-cell lung cancer," PLoS One, vol. 8, no. 12, Article ID e82241, 2013.

[18] T. Li, J. Fan, B. Wang et al., "TIMER: a web server for comprehensive analysis of tumor-infiltrating immune cells," Cancer Research, vol. 77, no. 21, pp. e108-e110, 2017.

[19] D. Sarrouilhe and M. Mesnil, "Serotonin and human cancer: a critical view," Biochimie, vol. 161, pp. 46-50, 2019.

[20] M. A. Peters, A. M. Walenkamp, I. P. Kema, C. Meijer, E. G. de Vries, and S. F. Oosting, "Dopamine and serotonin regulate tumor behavior by affecting angiogenesis," Drug Resistance Updates: Reviews and Commentaries in Antimicrobial and Anticancer Chemotherapy, vol. 17, no. 4-6, pp. 96-104, 2014.

[21] N. Dizeyi, P. Hedlund, A. Bjartell, M. Tinzl, K. Austild-Taskén, and P.-A. Abrahamsson, "Serotonin activates MAP kinase and PI3K/Akt signaling pathways in prostate cancer cell lines," Urologic Oncology: Seminars and Original Investigations, vol. 29, no. 4, pp. 436-445, 2011.

[22] N. Dizeyi, A. Bjartell, E. Nilsson et al., "Expression of serotonin receptors and role of serotonin in human prostate cancer tissue and cell lines," The Prostate, vol. 59, no. 3, pp. 328-336, 2004.

[23] Q. Wang, H. Zhang, H. Xu et al., "5-HTR3 and 5-HTR4 located on the mitochondrial membrane and functionally regulated mitochondrial functions," Scientific Reports, vol. 6, no. 1, Article ID 37336, 2016.

[24] M. Yarchoan, A. Hopkins, and E. M. Jaffee, "Tumor mutational burden and response rate to PD-1 inhibition," New England Journal of Medicine, vol. 377, no. 25, pp. 2500-2501, 2017.

[25] R. M. Samstein, C.-H. Lee, A. N. Shoushtari et al., "Tumor mutational load predicts survival after immunotherapy across multiple cancer types," Nature Genetics, vol. 51, no. 2, pp. 202-206, 2019.

[26] Z. Wang, J. Zhao, G. Wang et al., "Comutations in DNA damage response pathways serve as potential biomarkers for immune checkpoint blockade," Cancer Research, vol. 78, no. 22, pp. 6486-6496, 2018.

[27] D. T. Le, J. N. Durham, K. N. Smith et al., "Mismatch repair deficiency predicts response of solid tumors to PD-1 blockade," Science, vol. 357, no. 6349, pp. 409-413, 2017.

[28] Z.-Y. Dong, W.-Z. Zhong, X.-C. Zhang et al., "Potential predictive value of TP53 and KRAS mutation status for response to PD-1 blockade immunotherapy in lung adenocarcinoma," Clinical Cancer Research, vol. 23, no. 12, pp. 3012-3024, 2017.

[29] Q. Jia, J. Wang, N. He, J. He, and B. Zhu, "Titin mutation associated with responsiveness to checkpoint blockades in solid tumors," JCI Insight, vol. 4, no. 10, 2019. 